\title{
Added Value of Contrast Medium in Whole-Body Hybrid Positron Emission Tomography/Magnetic Resonance Imaging: Comparison between Contrast-Enhanced and Non-Contrast-Enhanced Protocols
}

\author{
Filiz Celebi $^{\mathrm{a}}$ Emetullah Cindil $^{\mathrm{b}}$ Dauren Sarsenov $^{\mathrm{c}}$ Bulent Unalan ${ }^{\mathrm{d}}$ \\ Cem Balcl ${ }^{e}$ \\ ${ }^{a}$ Department of Radiology, Gayrettepe Florence Nightingale Hospital, Istanbul, Turkey; ${ }^{\mathrm{b}}$ Department of Radiology, \\ Gazi University School of Medicine, Istanbul, Turkey; ' Department of General Surgery, Florence Nightingale

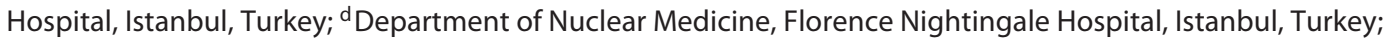 \\ e Department of Radiology, Cleveland Clinic, Lerner School of Medicine, Abu Dhabi, United Arab Emirates
}

\section{Highlights}

- A comprehensive oncologic imaging is achieved with PET/MRI when an appropriate protocol is used.

- One single examination can cover all organ systems and may detect all types of malignancies.

- A shorter and more accurate Whole Body PET/MRI protocol can be developed for the evaluation of malignancies.

\section{Keywords}

Positron emission tomography/magnetic resonance imaging · Malignancy · Fast protocol · Contrast medium

\footnotetext{
Abstract

Objective: To compare the diagnostic ability and time efficiency of contrast-enhanced (CE) whole body fluorodeoxyglucose (FDG) positron emission tomography/magnetic resonance imaging (PET/MRI) protocol and non-CE (NCE) protocol. Subjects and Methods: Ninety-three patients with known primary tumors underwent whole-body hybrid FDG $\mathrm{PET} / \mathrm{MRI}$ during the follow-up of their malignancies with the use of NCE and CE protocols. The NCE PET/MRI protocol con-
}

\begin{tabular}{ll}
\hline KARGER & $\begin{array}{l}\text { ( 2019 The Author(s) Karger } \\
\text { Published by S. Karger AG, Basel }\end{array}$ \\
E-Mail karger@karger.com & $\begin{array}{l}\text { This is an Open Access article licensed under the Creative Commons } \\
\text { Attribution-NonCommercial-4.0 International License (CC BY-NC) } \\
\text { (http://www.karger.com/Services/OpenAccessLicense), applicable to } \\
\text { the online version of the article only. Usage and distribution for com- } \\
\text { mercial purposes requires written permission. }\end{array}$
\end{tabular}

sisted of diffusion-weighted ( $b=0$ and $800 \mathrm{~s} / \mathrm{mm}^{2}$ ) and T1weighted Turbo Flash in the axial plane and T2-weighted HASTE sequence in the coronal planes ( $\Sigma=25 \mathrm{~min}$ ). The CE $\mathrm{PET} / \mathrm{MRI}$ protocol was performed by acquiring axial serial $\mathrm{CE}$ 3D FS VIBE images in the upper abdomen, completing the whole body in the late phase in the axial plane ( $\Sigma=30 \mathrm{~min}$ ). Results: There was a statistically significant difference between the total number of lesions detected by the CE protocol (median 2, interquartile range (IQR) 0-14) and that detected by the NCE protocol (median 1, IQR 0-5; $p<0.001$ ). More malignancies were detected in the abdomen $(p<$ $0.001)$ and brain $(p<0.001)$ with the CE PET/MRI protocol, whereas no significant difference was present when comparing the 2 protocols in the detection of malignancies in the 
head and neck ( $p=0.356)$, thorax $(p=0.09)$, lymph nodes $(p=0.196)$, and bone $(p=0.414)$. Conclusion: The CE FDG $\mathrm{PET} / \mathrm{MRI}$ protocol enables fast and accurate detection of malignancies compared to the NCE FDG PET/MRI protocol, particularly in the upper abdomen and brain. Diagnostic ability and time efficiency can be increased with the proposed short CE protocol in place of the whole body PET/MRI protocol including both NCE and CE imaging sequences.

\section{(c) 2019 The Author(s)}

Published by S. Karger AG, Basel

\section{Introduction}

Hybrid positron emission tomography/magnetic resonance imaging (PET/MRI) scanners have the potential to become an effective tool for the evaluation of oncology patients before, during, and after treatment and can influence patient management $[1,2]$. Hybrid PET/MRI uses PET data to assess the metabolic information of malignant tumors and relies on the uptake of radiotracers. However, certain tumors such as mucinous carcinoma or signet cell-type adenocarcinoma may not reveal the uptake of fluorodeoxyglucose (FDG), which is the most commonly used radiotracer. On the other hand, the physiological uptake of FDG in the liver and brain may prevent the depiction of tumors. Therefore, MRI data collected from hybrid PET/MRI systems not only provide superior soft tissue contrast and anatomic detail but may also improve the evaluation of malignant tumors. Therefore, the choice of imaging protocol is crucial for increasing the sensitivity of MRI. The use of intravenous contrast media is essential to oncologic imaging, especially for the detection of malignant tumors in the brain and in the upper abdomen [3-5]. In this study, we investigated whether contrast-enhanced (CE) whole-body $\mathrm{PET} / \mathrm{MRI}$ protocols increase the sensitivity of detection of malignant tumors compared to non-CE (NCE) protocols.

\section{Subjects and Methods}

In our study, 93 consecutive patients with histopathologically proven primary malignant tumors aged between 20 and 87 were retrospectively evaluated (mean age \pm SD, $55.1 \pm 14.1$ years) during the period of August 2015 to January 2018. Fifty-four of these patients were men (53.4 \pm 14.7 years), and 39 of them were women (56.4 \pm 13.6 years). The institutional review board approved the study; the requirement of informed consent was waived, since the study was a retrospective investigation. The patients referred to PET/MRI from the hematology/oncology and surgery depart- ments as part of the standard oncology imaging were included in the study. Patients with a history of allergy to gadolinium-based contrast agents and end-stage renal failure were excluded from the study. All patients had histopathological proof of their primary malignant tumors by surgery $(n=56)$ and/or biopsy $(n=37)$.

Metastatic lesions without histopathologic evidence were identified based on the progression of their size or the appearance of new lesions during follow-up. The malignancy of the lymph nodes was determined based on their size and/or FDG uptake.

Eight patients underwent neoadjuvant therapy, and PET/MRI was performed at follow-up; 45 patients had their primary tumor surgically removed, followed by adjuvant chemotherapy and/or chemoradiotherapy. In 40 patients, the primary tumor was not deemed operable according to the criteria of other imaging modalities, and these patients either underwent chemotherapy and/or chemoradiotherapy.

All patients fasted for at least $6 \mathrm{~h}$ before imaging. The blood glucose level was assessed with a blood glucose meter (OneTouch Vita; LifeScan, Milpitas, CA, USA) before imaging to ensure that it was $<140 \mathrm{mg} / \mathrm{dL}(7.77 \mathrm{mmol} / \mathrm{L})$.

PET/MRI was performed $45 \pm 10 \mathrm{~min}$ after the injection of FDG (mean dose, $4.54 \mathrm{MBq}$ per kilogram of body weight \pm 1 ; range, $370-400 \mathrm{MBq}$ ). The images were acquired in supine position on a 3 Tesla Biograph mMR scanner (Siemens Healthcare, Erlangen, Germany) using a 16-channel head and neck surface coil and three 12-channel body coils. These body coils were combined to form a multichannel whole-body coil by using the total imaging matrix technology. The whole-body images were obtained in 5 to 6 bed positions according to the size of the patient and each bed time position was maintained between 2 and $2.5 \mathrm{~min}$. PET acquisition occurred simultaneously during the whole-body MRI acquisition. In all patients, the whole-body PET/MRI covered the entire body from head to knee. For the attenuation correction, 4-point Dixon images were obtained in the coronal plane. The whole-body MRI protocol consisted of both NCE images and CE images and provided the most comprehensive oncologic imaging dataset in the routine in our institution. The imaging stations were arranged consecutively in the caudocranial direction. The comprehensive MRI protocol that included both CE and NCE studies consisted of T2-weighted single-shot echo train (HASTE; TR/TE, 1,500 ms/87 $\mathrm{ms}$ ) in the coronal plane, T1-weighted slice-selective Turbo Flash (TR/TE, $1,600 \mathrm{~ms} / 2.5 \mathrm{~ms}$ ), and free breath diffusion-weighted imaging (DWI) using EPI technique (TR/TE, $12,000 \mathrm{~ms} / 78 \mathrm{~ms}, \mathrm{~b}=0$ and $800 \mathrm{~s} / \mathrm{mm}^{2}$ ) in the axial planes. After the NCE protocol was performed, a weight-adapted dose of a gadolinium-based contrast agent was administered, and serial CE images were obtained using breath-hold 3D VIBE (TR/TE, $4.56 \mathrm{~ms} / 2.03 \mathrm{~ms})$ in the arterial, portal venous and equilibrium phases covering the upper abdomen in the axial plane. After the serial CE images were acquired, continuous breath-hold 3D VIBE images were obtained from head to knee in the axial plane, and all the sections were combined, resulting in uninterrupted whole-body coverage. Detailed information regarding the whole-body PET/MRI protocol is shown in Table 1. The total scan duration of the PET/MRI examination was 50-60 min. The durations of both the NCE and CE protocols were $25-30 \mathrm{~min}$.

The images were evaluated by 2 radiologists; one had 25 years of experience, and the other had 8 years of experience in reading MRI and hybrid imaging. Both readers were blinded to patient data and diagnosis. One research associate separated each study 
into 2 parts, and the loaded images had an NCE part and a CE part. Data were analyzed on a dedicated workstation (Syngo Via; Siemens Healthcare, Erlangen, Germany). The NCE and CE protocols were reviewed 1 month apart to avoid bias. The review of the cases for CE and NCE studies took $1 \mathrm{~h}$ each. All images with diagnostic quality for PET and MRI were evaluated separately and as fused images, the number of lesions was recorded for both protocols. The radiologists and a nuclear medicine physician evaluated the images in a joint reading session and made consensus. The NCE dataset was evaluated using NCE MR images, PET/MRI fusion images, and attenuation-corrected raw data PET images. The CE dataset was evaluated by reviewing CE MR images, fused PET/MRI images, and attenuation-corrected raw data PET images.

Statistical analyses were performed using SPSS software version 20 . The variables were investigated using visual methods (histograms, probability plots) and analytical methods (Kolmogorov-Smirnov) to determine whether or not the distribution was normal.

As most of the variables excepting "age" were not represented by real numbers, descriptive analyses for tests were presented using medians and interquartile range (IQR). Friedman's test was conducted to evaluate whether there is a significant change in the total number of detected lesions among different MRI sequences separately for each anatomic region (due to violations of parametric test assumptions as number of lesions detected can only be integer). Pairwise comparisons were performed using Wilcoxon signed rank test. A $p$ value of $\leq 0.05$ was accepted as statistically significant.

\section{Results}

Of the 93 patients, 17 patients had non FDG-avid malignant tumors. The rest of the patients had FDG avid malignant tumors, 36 patients had distance metastasis $(\mathrm{M})$, and 40 patients had lymph node metastases $(\mathrm{N})$.

Distribution of the primary malignant tumors is shown in Table 2. Gastrointestinal tumors include gastric, pancreatic, colorectal cancers, hepatocellular carcinoma, and cholangiocarcinoma.

The number of lesions detected using the CE protocol (median 2, IQR 0-14) was significantly higher than that detected using the NCE protocol (median 1, IQR 0-5; $p<$ 0.001 ). The total number of lesions detected using the $\mathrm{CE}$ protocol varied between 0 and 120 (minimum-maximum), whereas that detected using the NCE protocol was 0-75 (minimum-maximum). The total number of lesions detected with only the PET component (median 1, IQR $0-11$ ) was significantly lower than the number of lesions detected with the CE PET/MR protocol (median 2, IQR $0-14 ; p<0.001$ ).

Regarding the number of region-based lesions, the number of lesions in the brain detected with the CE pro-

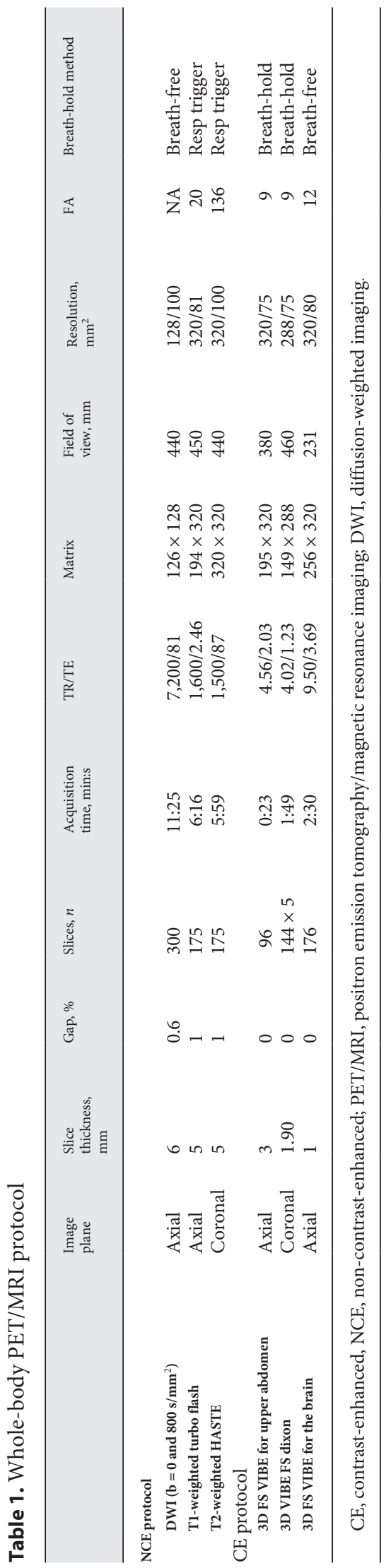

Celebi/Cindil/Sarsenov/Unalan/Balc1 
Fig. 1. A 56-year-old patient with chronic liver disease. An enhancing nodule is present in segment 6 of the liver in the arterial phase (arrow, a) that reveals contrast washout on the portal venous phase (arrow, b) during the CE PET/MRI protocol. On the diffusion-weighted MRI (c) and PET/MRI fusion images (d), no lesion is visible during the NCE PET/MRI protocol.
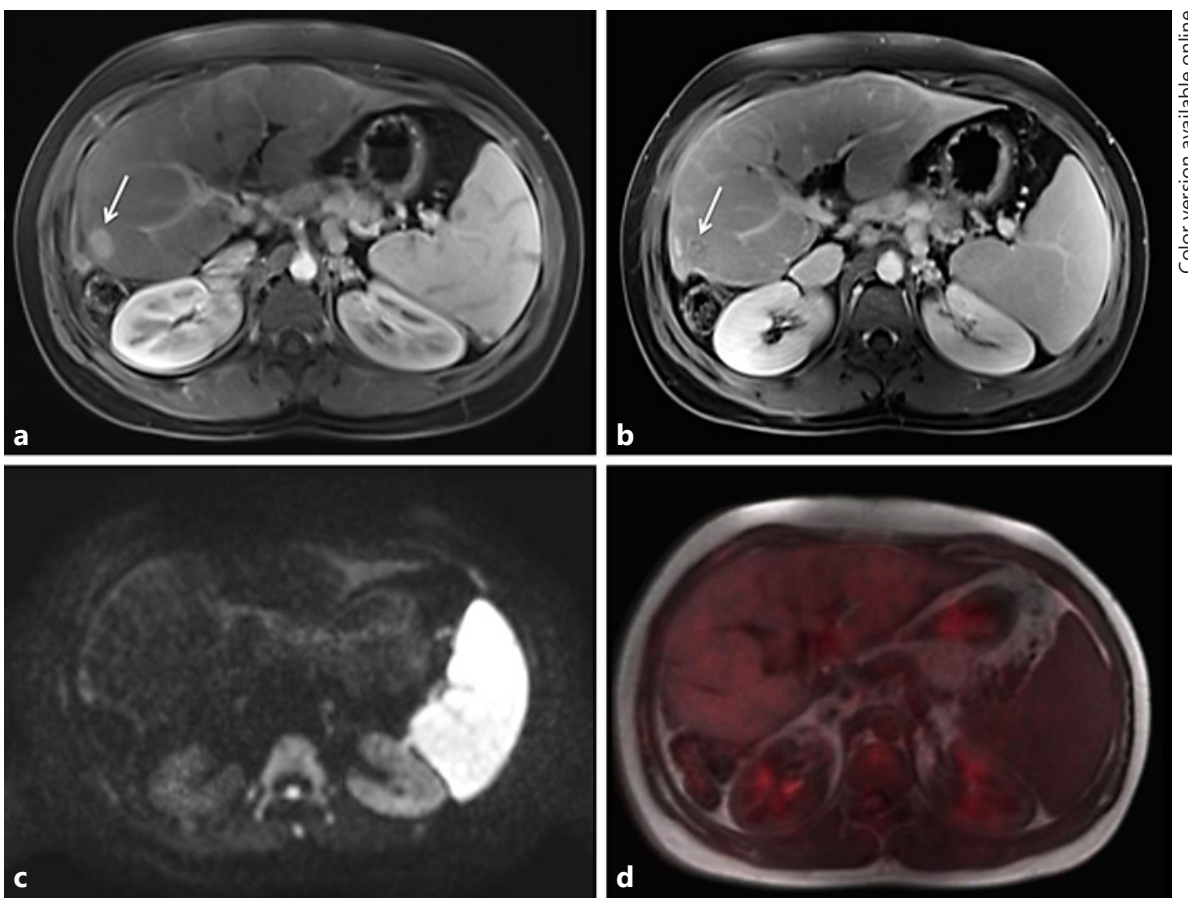

Table 2. Distribution of primary malignant tumors

\begin{tabular}{ll}
\hline Primary malignant tumors & $\begin{array}{l}\text { Number of } \\
\text { patients }\end{array}$ \\
\hline Hepatobiliary & 28 \\
Other gastrointestinal & 16 \\
Genitourinary & 10 \\
Breast & 18 \\
Immunoproliferative & 6 \\
Lung carcinoma & 6 \\
Other & 9 \\
\hline
\end{tabular}

Table 3. Distribution of malignant tumors for different body regions

\begin{tabular}{lllllll}
\hline \multirow{2}{*}{ Region } & \multicolumn{2}{l}{ CE protocol } & & \multicolumn{2}{l}{ NCE protocol } \\
\cline { 2 - 3 } \cline { 6 - 7 } & median & IQR & & median & IQR & $p$ \\
\hline Brain & 2 & $1-2$ & & 0 & $0-1$ & $<0.001$ \\
Head and neck & 2 & $1-3.5$ & & $1-4$ & 0.356 \\
LN & 1 & $1-4$ & & 1 & $1-3$ & 0.196 \\
Thorax & 1 & $1-4$ & & 1 & $1-3$ & 0.09 \\
Abdomen & 2 & $1-6$ & & 1 & $0-2$ & $<0.001$ \\
Bone & 5 & $1-30$ & & 4.5 & $1-30$ & 0.414 \\
Total & 2 & $0-14$ & & 1 & $0-5$ & $<0.001$ \\
\hline
\end{tabular}

Friedman's test.

Whole Body Hybrid PET/MRI tocol (median 2, IQR 1-2) was significantly higher than that detected with the NCE protocol (median 0, IQR 0-1; $p=0.001, n=11$; Fig. 1 ).

In the abdomen, the CE PET/MRI protocol (median 2, IQR 1-6) was superior to the NCE protocol (median1, IQR 0-2; $p<0.001, n=65$; Fig. 2).

There was no difference between the number of malignant tumors detected by the 2 protocols for the head and neck $(p=0.356, n=13)$, bone $(p=0.414, n=28)$, thorax $(p=0.09, n=31$; Fig. 3$)$, and lymph nodes $(p=0.196, n=$ 32; Table 3).

\section{Discussion}

Our study showed that the CE fast PET/MRI protocol depicted more malignant tumors in the upper abdomen and in the brain compared to the NCE protocol. In addition, our CE fast protocol may increase patient comfort due to its short duration and is an effective tool for the evaluation of oncology patients before, during, and after treatment.

PET/MRI is a new imaging modality that combines the sensitivity of molecular imaging of PET and the superior radiologic diagnostic capabilities of MRI. In addition, PET/MRI provides detailed background anatomical landmarks from MRI images. MRI also bestows superior 

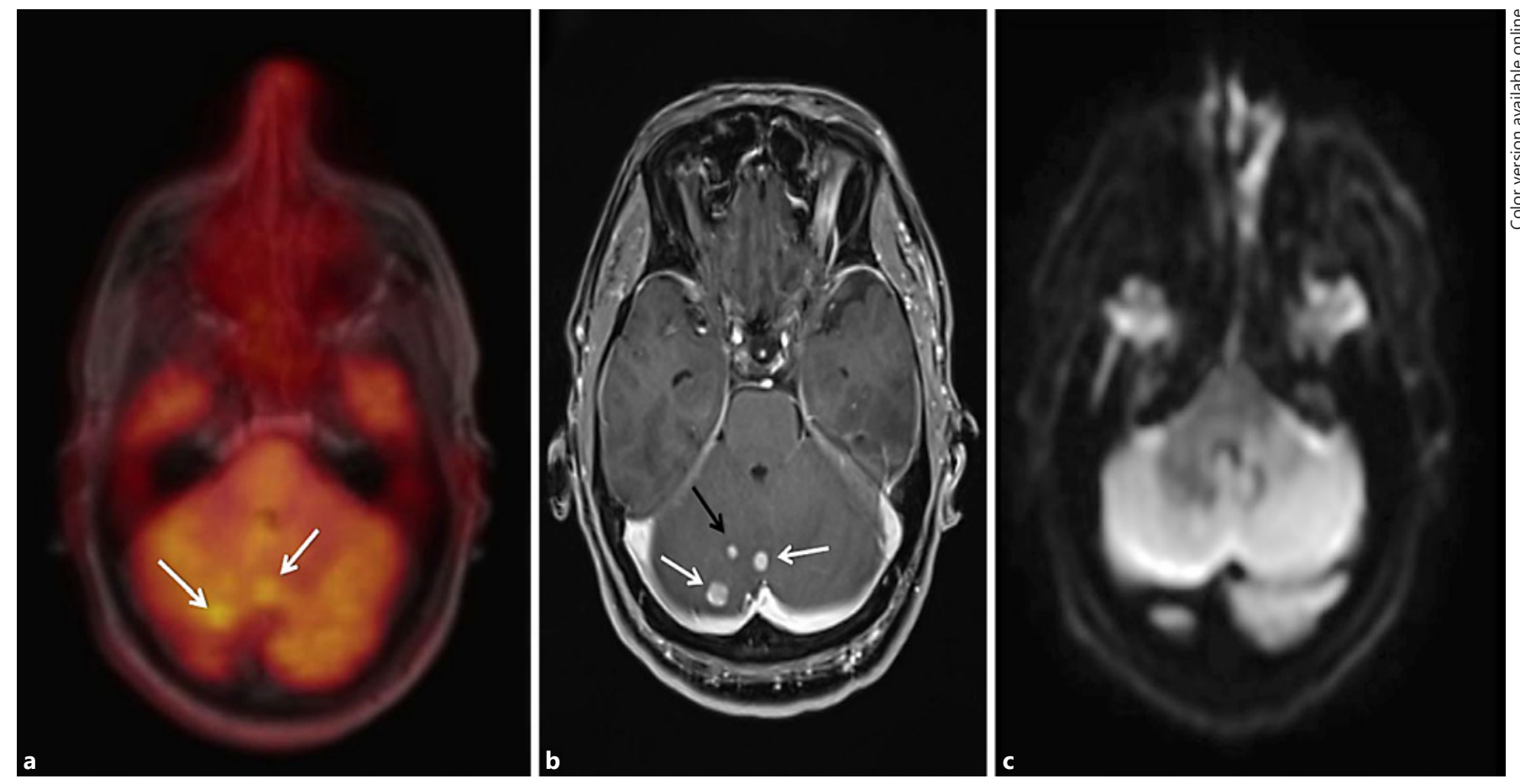

Fig. 2. A 67-year-old patient with breast cancer. NCE PET/MRI fusion images reveal 2 FDG-avid lesions in the cerebellum (arrows, a) that are not seen on the diffusion-weighted MRI (b). 3D VIBE postcontrast images acquired using the CE PET/MRI protocol reveal both metastases (white arrows, $\mathbf{c}$ ) and an additional smaller lesion (black arrow, c).
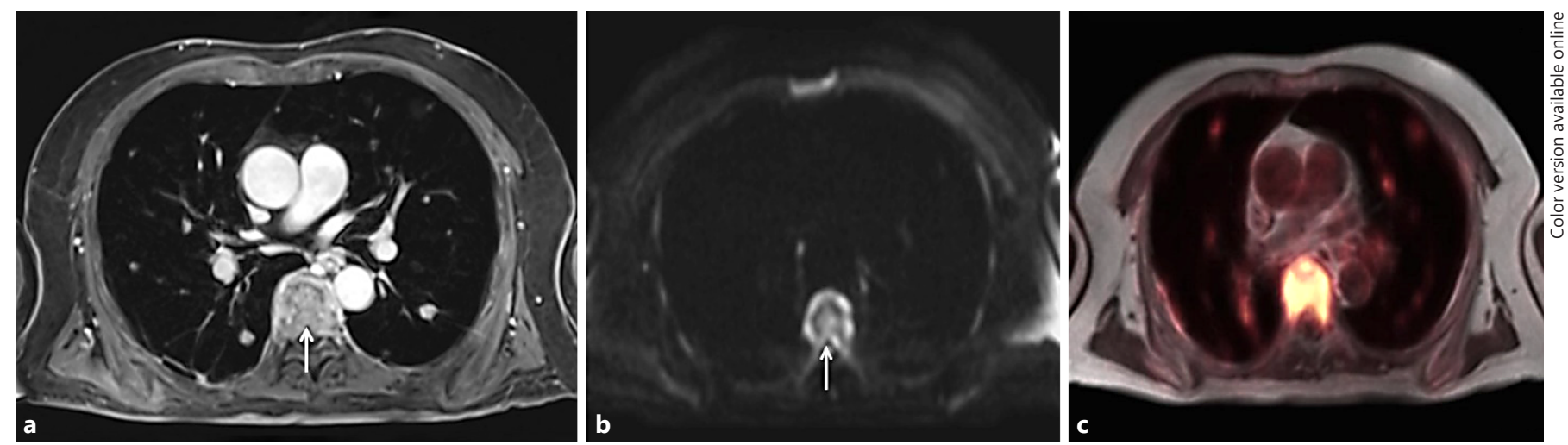

Fig. 3. A 72-year-old patient with advanced colon cancer. CE PET/MRI protocol reveals multiple lung metastases and bone involvement in the thoracic vertebra (arrow, a) on the postcontrast 3D VIBE axial image. NCE protocol with diffusion-weighted MRI reveals bone involvement (arrow, b) with less apparent lung lesions. Fusion $\mathrm{PET} / \mathrm{MRI}$ images of the non-contrast protocol depicts both lung and bone metastases (arrow, c).

tissue contrast that helps to localize tumors and assist in the local staging (T staging) of tumors. PET/MR is superior to PET/CT because its resolves soft tissue without the need for radiation exposure. The metabolic information from PET data together with the diagnostic accuracy of
CE whole-body MRI may increase the sensitivity of tumor detection.

Several studies have shown that the detection of primary and metastatic liver and brain tumors by MRI is superior to that by PET $[3,5]$. In liver imaging, serial CE 
images are essential to depict hypervascular lesions, and only CE images can depict brain metastases [3, 4, 6-9]. In the pancreas, $\mathrm{CE}$ images may depict hypervascular neuroendocrine tumors that are not FDG avid [10-12]. Additionally, renal tumors may be evaluated with CE images to differentiate between benign and malignant lesions [13]. PET images rely on the uptake of FDG, which is taken up physiologically by the tissues of the liver and brain. Primary liver tumors such as HCC can only take up FDG if they are less differentiated $[14,15]$. In our dataset, CE PET/MRI depicted more HCC with the serial CE images. HCC is a hypervascular tumor with arterial blood supply, and arterial enhancement with venous washout is diagnostic for HCC in serial CE images [8].

In contrast to our results, Lee at al. [16] presented that the effect of gadolinium-based contrast agents on PET image can be negligible quantitatively and qualitatively. In imaging renal and bladder tumors, MRI is superior to PET with CE protocols. Renal lesions, including cysts, can only be categorized according to their enhancement pattern [17]. In our dataset, we did not observe any primary renal tumor; however, CE MRI protocols can further help to distinguish between malignant and benign renal lesions. We did not observe any significant difference in bone metastasis and lymph node involvement.

FDG-PET detection of hypometabolic metastasis and increased bone marrow activity after chemotherapy is not sensitive [18-20]. Bone marrow-sensitive MRI techniques can provide diagnostic information on FDG-negative cases [21]. Our data showed that the combined evaluation of PET and MRI with either DWI or post-contrast VIBE equally assessed the bone marrow metastases. Previous studies have shown that CE MRI and DWI have similar sensitivities for the detection of bone metastases $[22,23]$.

Stolzman et al. [24] and Catalano et al. [25] compared lung nodule detection rates using CT, MR, and PET either in different combinations or separately. They found similar detection rates for both PET/CT and PET/MRI. Our study revealed similar results for the detection of lung nodules. The duration of the whole-body PET/MRI ex- amination is long, and it is unpleasant for the patient to undergo a 1-h examination in a closed environment. Therefore, shorter protocols have been performed [26, 27]. Our CE protocol may be used as a standard protocol for shorter examinations, which may increase patient throughput and patient comfort.

The limitation of our study may include the limited number of cases of each malignancy group and see which particular malignancy may benefit the most for CE protocol. Also, not all cases were histopathologically proven. Further prospective studies may be needed for tailored PET/MRI protocols for specific malignancies especially in terms of local staging. CE studies may be performed with the use of improved temporal resolution to calculate the contrast passage (Ktrans) and monitor the antiangiogenic treatments.

\section{Conclusion}

An optimal oncologic imaging was achieved using the appropriate protocol for PET/MRI, and a variety of tumor types and involvement of the organ systems can be reviewed by optimizing the protocol. We investigated the use of the CE whole-body PET/MRI protocol for the assessment of malignant tumors, and our results indicated that MRI with intravenous contrast might increase the sensitivity of PET/MRI. The protocol with the emphasis of $\mathrm{CE}$ examination can further shorten the PET/MRI exam with increased detection rate of neoplasms.

\section{Statement of Ethics}

This study was performed according to the Declaration of Helsinki on medical protocols and was approved by the Bilim University Institutional Review Board (No. 28.06.2016/51-06).

\section{Disclosure Statement}

The authors have no conflicts of interest to declare.

\section{References}

Whole Body Hybrid PET/MRI
1 Kang B, Lee JM, Song YS, Woo S, Hur BY, Jeon $\mathrm{JH}$, et al. Added value of integrated whole-body PET/MRI for evaluation of colorectal cancer: comparison with contrastenhanced MDCT. AJR Am J Roentgenol. 2016 Jan;206(1):W10-20.

2 Torigian DA, Zaidi H, Kwee TC, Saboury B, Udupa JK, Cho ZH, et al. PET/MR imaging: technical aspects and potential clinical applications. Radiology. 2013 Apr;267(1):26-44.

3 Kitajima K, Nakamoto Y, Okizuka H, Onishi Y, Senda M, Suganuma N, et al. Accuracy of whole-body FDG-PET/CT for detecting brain metastases from non-central nervous system tumors. Ann Nucl Med. 2008 Aug; 22(7):595-602. 
4 Lohmann P, Stoffels G, Ceccon G, Rapp M, Sabel M, Filss CP, et al. Radiation injury vs. recurrent brain metastasis: combining textural feature radiomics analysis and standard parameters may increase 18F-FET PET accuracy without dynamic scans. Eur Radiol. 2017 Jul;27(7):2916-27.

5 Niekel MC, Bipat S, Stoker J. Diagnostic imaging of colorectal liver metastases with CT, MR imaging, FDG PET, and/or FDG PET/ CT: a meta-analysis of prospective studies including patients who have not previously undergone treatment. Radiology. 2010 Dec; 257(3):674-84.

6 Lee JM, Zech CJ, Bolondi L, Jonas E, Kim MJ, Matsui $\mathrm{O}$, et al. Consensus report of the 4th International Forum for Gadolinium-Ethoxybenzyl-Diethylenetriamine Pentaacetic Acid Magnetic Resonance Imaging. Korean J Radiol. 2011 Jul-Aug;12(4):403-15.

7 Lee KH, Lee JM, Park JH, Kim JH, Park HS, $\mathrm{Yu} \mathrm{MH}$, et al. MR imaging in patients with suspected liver metastases: value of liver-specific contrast agent gadoxetic acid. Korean J Radiol. 2013 Nov-Dec;14(6):894-904.

8 Di Martino M, Marin D, Guerrisi A, Baski M, Galati F, Rossi M, et al. Intraindividual comparison of gadoxetate disodium-enhanced MR imaging and 64-section multidetector CT in the Detection of hepatocellular carcinoma in patients with cirrhosis. Radiology. 2010 Sep;256(3):806-16.

9 Seo HJ, Kim MJ, Lee JD, Chung WS, Kim YE. Gadoxetate disodium-enhanced magnetic resonance imaging versus contrast-enhanced 18F-fluorodeoxyglucose positron emission tomography/computed tomography for the detection of colorectal liver metastases. Invest Radiol. 2011 Sep;46(9):548-55.

10 Baumann T, Rottenburger C, Nicolas G, Wild D. Gastroenteropancreatic neuroendocrine tumours (GEP-NET) - Imaging and staging. Best Pract Res Clin Endocrinol Metab. 2016 Jan;30(1):45-57.

11 Panagiotidis E, Alshammari A, Michopoulou S, Skoura E, Naik K, Maragkoudakis E, et al.
Comparison of the impact of 68Ga-DOTATATE and 18F-FDG PET/CT on clinical management in patients with neuroendocrine tumors. J Nucl Med. 2017 Jan;58(1):91-6.

12 Dromain C, Déandréis D, Scoazec JY, Goere D, Ducreux M, Baudin E, et al. Imaging of neuroendocrine tumors of the pancreas. $\mathrm{Di}$ agn Interv Imaging. 2016 Dec;97(12):124157.

13 Vargas HA, Chaim J, Lefkowitz RA, Lakhman Y, Zheng J, Moskowitz CS, et al. Renal cortical tumors: use of multiphasic contrast-enhanced MR imaging to differentiate benign and malignant histologic subtypes. Radiology. 2012 Sep;264(3):779-88.

14 Takeuchi S, Rohren EM, Abdel-Wahab R, Xiao L, Morris JS, Macapinlac HA, et al. Refining prognosis in patients with hepatocellular carcinoma through incorporation of metabolic imaging biomarkers. Eur J Nucl Med Mol Imaging. 2017 Jun;44(6): 969-78.

15 Torizuka T, Tamaki N, Inokuma T, Magata Y, Sasayama S, Yonekura Y, et al. In vivo assessment of glucose metabolism in hepatocellular carcinoma with FDG-PET. J Nucl Med. 1995 Oct;36(10):1811-7.

16 Lee WH, Park JA, Kim KY. Effects of MR contrast agents on PET quantitation in PET-MRI study. J Nucl Med. 2011 May;52 Suppl 1:53.

17 Verma S, Rajesh A, Prasad SR, Gaitonde K, Lall CG, Mouraviev V, et al. Urinary bladder cancer: role of MR imaging. Radiographics. 2012 Mar-Apr;32(2):371-87.

18 Nakai T, Okuyama C, Kubota T, Yamada K, Ushijima Y, Taniike K, et al. Pitfalls of FDGPET for the diagnosis of osteoblastic bone metastases in patients with breast cancer. Eur J Nucl Med Mol Imaging. 2005 Nov;32(11): 1253-8.

19 Hamaoka T, Madewell JE, Podoloff DA, Hortobagyi GN, Ueno NT. Bone imaging in metastatic breast cancer. J Clin Oncol. 2004 Jul; 22(14):2942-53.

20 Sugawara Y, Fisher SJ, Zasadny KR, Kison PV, Baker LH, Wahl RL. Preclinical and clinical studies of bone marrow uptake of fluorine1-fluorodeoxyglucose with or without granulocyte colony-stimulating factor during chemotherapy. J Clin Oncol. 1998 Jan;16(1):17380.

21 Schmidt GP, Reiser MF, Baur-Melnyk A. Whole-body imaging of the musculoskeletal system: the value of MR imaging. Skeletal Radiol. 2007 Dec;36(12):1109-19.

22 Soliman M, Taunk NK, Simons RE, Osborne JR, Kim MM, Szerlip NJ, et al. Anatomic and functional imaging in the diagnosis of spine metastases and response assessment after spine radiosurgery. Neurosurg Focus. 2017 Jan;42(1):E5.

23 Lecouvet FE, Larbi A, Pasoglou V, Omoumi $\mathrm{P}$, Tombal B, Michoux N, et al. MRI for response assessment in metastatic bone disease. Eur Radiol. 2013 Jul;23(7):1986-97.

24 Stolzmann P, Veit-Haibach P, Chuck N, Rossi C, Frauenfelder T, Alkadhi H, et al. Detection rate, location, and size of pulmonary nodules in trimodality PET/CT-MR: comparison of low-dose CT and Dixon-based MR imaging. Invest Radiol. 2013 May;48(5):2416.

25 Catalano OA, Rosen BR, Sahani DV, Hahn PF, Guimaraes AR, Vangel MG, et al. Clinical impact of PET/MR imaging in patients with cancer undergoing same-day PET/CT: initial experience in 134 patients-a hypothesisgenerating exploratory study. Radiology. 2013 Dec;269(3):857-69.

26 Grueneisen J, Sawicki LM, Schaarschmidt $\mathrm{BM}$, Suntharalingam S, von der Ropp S, Wetter A, et al. Evaluation of a fast protocol for staging lymphoma patients with integrated PET/MRI. PLoS One. 2016 Jun; 11(6): $\mathrm{e} 0157880$.

27 Grueneisen J, Schaarschmidt BM, Heubner M, Suntharalingam S, Milk I, Kinner S, et al. Implementation of FAST-PET/MRI for whole-body staging of female patients with recurrent pelvic malignancies: A comparison to PET/CT. Eur J Radiol. 2015 Nov;84(11): 2097-102. 